\title{
Lecturas etnográficas para los sistemas de innovación local*
}

\author{
Ethnographic readings for local innovation systems
}

\author{
Claudio Gajardo Cortés* \\ Francisco Ther Ríos ${ }^{* 2}$
}

Palabras-

claves:

Sistemas de Innovación local;

Conocimiento local y científico;

Situaciones

Prácticas;

Sociedades

Litorales; Isla de Chiloé.

\begin{abstract}
Resumen: Las prácticas productivas de los pescadores artesanales portan habilidades y conocimientos cuyas combinaciones singularizan, desde su diferencia, las nociones de innovación y tecnología implícitas en las perspectivas del desarrollo. Algunas situaciones prácticas donde coexisten conocimientos locales y científicos informan lo anterior desde su participación en la construcción sociocultural del ambiente local. El presente trabajo describe tres situaciones en el sector de Guabún, zona noroccidental de la Isla de Chiloé; i) La técnica para subir desde el fondo del mar a la superficie por los buzos mariscadores de Luga (Gigartina skottsbergii), ii) Las prácticas en un Área de Manejo y Explotación de Recursos Bentónicos (AMERB) de la especie Loco (Concholepas concholepas) y iii) La extracción de Algas por recolectoras y buzos mariscadores habitantes del lugar. Al considerar simétricamente estos tipos de conocimientos, la innovación local puede revisarse a la luz de la experiencia compleja de cada ambiente local. Se apuesta con ello a reconsiderar las singularidades socio productivas de las caletas de pescadores como expresiones de la capacidad creativa de sus habitantes y a su potencialidad como posibilidad de alternativas de nuevo conocimiento para el desarrollo territorial de sociedades vinculadas a ecosistemas locales.
\end{abstract}

\footnotetext{
* Artículo recibido en 19/03/2013 y aceito para publicación en 18/06/2013. En este artículo se presentan parte de los avances logrados a través del Proyecto MEL 81100002 "Valoración Económico -Ambiental de las Actividades Pesqueras Artesanales. Estrategias de Diversificación Productiva en Sociedades Litorales del Sur de Chile",

${ }^{* 1}$ Antropólogo. Estudiante Tesista Magister en Ciencias Sociales, ULA; Investigador asociado Programa ATLAS / Universidad de los Lagos. E-mail: clgajardocortes@gmail.com.

*2 Antropólogo, director Programa ATLAS da Universidad de los Lagos, Chile. E-mail: fther@hotmail.com.
} 
Keywords: Abstract: The productive practices of artisanal fishermen include System of local innovation; Local and scientific knowledge;

Practical situations;

Coastal societies; Chiloé Island. abilities and knowledge; the combinations of these determine, based on their differences, the notions of innovation and technology implicit in the perspectives of their development. Some practical situations where local and scientific knowledge coexist corroborate this, given their participation in the socio-cultural construction of the local environment. The present work describes three situations in the sector of Guabún, in the northwest zone of the island of Chiloé: i) the technique used by Luga (Gigartina Skottsbergii) harvesters to rise from the bottom of the ocean to the surface; ii) the practices of Loco (Concholepas concholepas) in the Benthic Resources Management and Exploration Area (Área de Manejo y Explotación de Recursos Bentónicos - AMERB); and iii) The extraction of seaweed by collectors and various fishermen living in the area. By comparing these types of knowledge equally, local innovation may bring to light the complex experience of each environment. The ideal is to analyze, in each environment, the unique socio-productive characteristics of the fishermen's coves as a consequence of their inhabitants' creative capacity and the potential possibility of land development alternatives for societies linked to local ecosystems.

\section{Introducción}

$\mathrm{D}$ esde la década de los noventa, luego de un período de apertura económica y consecuentemente de sobreexplotación de recursos en el país (BUSTAMENTE y CASTILLA, 1987; CASTILLA, 1990; BUSTOS et al., 1991), las caletas de pescadores de la Isla de Chiloé han estado asimilando normativas, formas de comercio y prácticas productivas sujetas a un acelerado y caótico proceso modernizador (MORALES, 1986; PEÑA, 1996; IBÁÑEZ et al., 2001; NEIRA, 2005; MARIN, 2007). Una de sus principales características ha sido el contacto con actores portadores de otros conocimientos con asociación al comercio, tales como científicos y administrativos (MARIN Y BERKES, 2010). Como resultado, los sistemas de prácticas locales enfrentan la introducción de conocimientos de distinto origen, cuyos riesgos, implícitos a la desorientación que distintas racionalidades producen en un mismo espacio geográfico, quedan asociados principalmente a i) la descontextualización de las particularidades locales (GIDDENS, 1991; HORNBORG, 1996), ii) el aislamiento y pérdida de la capacidad de reinvención frente a contextos emergentes (NANDY, 1988; VESSURI, 2004) y iii) la dificultad para elaborar una construcción social de 
futuro donde se garantice su reproducción económica, ecológica y cultural (LEFF, 2001, 2002a, 2002b, 2006).

Actualmente, en las prácticas de recolección de orilla, en las formas de pesca mar adentro o en las técnicas de buceo en profundidad, es posible observar al menos un componente científico y uno local (THER RÍOS, 2008). Esta coexistencia tiene lugar i) al considerar que las habilidades de los pescadores para desarrollarse en condiciones geográficas y ecosistémicas particulares incluyen un proceso de adaptación al ambiente y ii) que las normativas superpuestas sobre esos ambientes son elaboradas desde una mirada impositiva y desinformada de las múltiples experiencias del ambiente local (DIAZ, 2008; HENRÍQUEZ, 2010; CEBALLOS, 2010; VALDERRAMA, 2010; GAJARDO, 2011; ÁLVAREZ, 2011). Considerando lo anterior, más allá de la búsqueda de coincidencias que demuestren la veracidad de ambos tipos de conocimiento, se trata de plantear posibles vías para elaborar un conocimiento innovador que, incluyendo a los anteriores, revitalice y genere alternativas de desarrollo sustentable para las caletas de pescadores (THER RÍOS, 2008).

En el presente trabajo, se describe etnográficamente tres situaciones prácticas de conocimiento distinguiendo sus aspectos científicos y locales con el objetivo de evidenciar posibilidades creativas para un conocimiento de innovación local en sociedades litorales (MEJÍA, 2008). Con lo anterior, se argumenta que la innovación puede ser no solo la generación de condiciones de productividad económica sino más bien la construcción de un conocimiento vinculado que, en contrapartida al conocimiento especializado y disyuntivo (MORIN, 1998), establezca la necesidad de participación de los actores del sistema cultural de la pesca artesanal pudiendo ser adaptable a la complejidad de cada geografía local (OSTROM, 2000).

\section{Metodología}

La investigación utilizó un diseño cualitativo aplicado a dos fuentes de información, a) relatos de pescadores artesanales acerca de sus prácticas productivas, b) narraciones científicas sobre los ambientes en que estas se realizan. Con ello fue posible contrastar y triangular ambos modos de conocimientos en función de la búsqueda de una mirada interdisciplinaria que incluye el conocimiento local en cada situación (ARIAS, 2009). La recolección de información se realizó mediante i) observación etnográfica con estadía prolongada, ii) aplicación de entrevistas en profundidad y iii) revisión documental de documentos científicos y técnicos tomando como referente la información recolectada en terreno. La sistematización de 
información y el proceso de triangulación, realizados durante los años 2009, 2010 y 2011, permitieron identificar situaciones prácticas de conocimientos dentro del sistema de prácticas de un mismo ambiente local. La selección de un solo sector geográfico responde a la necesidad de ejemplificar la diversidad interna presente en las sociedades litorales.

Los pescadores entrevistados fueron buzos mariscadores y mujeres recolectoras de orilla habitantes del sector de Guabún, norte de la Isla Grande de Chiloé. La selección se realizó mediante la exploración en terreno siguiendo el método de la bola de nieve hasta lograr un criterio de saturación de información (EYLES, 1998; CANALES, 2006). Los informes técnicos y científicos fueron seleccionados de acuerdo a su referencia al sector y específicamente a las prácticas estudiadas. Las entrevistas, observaciones etnográficas y revisiones documentales se realizaron de acuerdo a los siguientes tópicos; i) espacios geográficos reducidos y delimitados, ii) técnicas y habilidades locales desplegadas sobre dichos espacios, iii) normativas y conocimientos operando sobre el espacio. Los tres tópicos constituyen la unidad de análisis que hemos denominado, situaciones prácticas de conocimientos. De ese modo, mediante el contraste de fuentes de información, la selección de situaciones responde a su relevancia en el uso narrativo por parte de los informantes y a las posibilidades de distinguir conocimientos científicos y locales, observadas por el investigador de acuerdo a la revisión documental y los antecedentes previos (JÓCILES, 1999).

Para establecer las diferencias teóricas entre ambos tipos de conocimientos se utilizó la perspectiva de la Etnoecología (REYES-GARCÍA y MARTÍ SANZ, 2007) y los Estudios Sociales de Ciencia y Tecnología (KREIMER, y THOMAS, 2004). Principalmente nos concentramos en sus características, diferencias y similitudes a partir de un registro etnográfico utilizado al modo de una descripción e interpretación de múltiples prácticas situacionales (DIAZ DE RADA, 2006). Estos contrastes se observaron siguiendo metodológicamente algunos aspectos de la antropología simétrica (LATOUR, 1993), principalmente con el objetivo de despejar el análisis de aquellas diferenciaciones que sitúan a un tipo de conocimiento por sobre el otro (LANDER, 2000).

La etnografía de situaciones prácticas de conocimiento busca indagar en la experiencia concreta del ambiente local, atendiendo a los siguientes aspectos; i) que las situaciones aparecen dentro de los contactos entre el investigador y los habitantes en el acontecer cotidiano en un territorio (THER RIOS, 2003, 2006), ii) que la interpretación asume la perspectiva de la antropología simétrica consistente en que, en lugar de subsumir las 
diferencias entre perspectivas desde una perspectiva universal, p. ej., la naturaleza biofísica, se supone en suspenso la predominancia de una mirada sobre otras posibles, permitiendo una aceptación más compleja y propositiva situada en las diferencias (LATOUR, 1993; VIVEIROS DE CASTRO, 2002; VILLORO, 2008), iii) que se capturan situaciones tanto en territorios distintos o en un mismo territorio, siguiendo más bien la dinámica de la circulación o combinación de conocimientos pudiendo ser, por tanto, una búsqueda etnográfica multisituada o multilocal (MARCUS, 2001) y iv) al trabajar en un mismo sector geográfico se aprecia el perspectivismo interno del territorio, considerando aquellas perspectivas bajo la noción del carácter hologramático del espacio (LINDÓN, 2006, 2007).

Finalmente, con la interpretación de las situaciones se manifiestan las potencialidades en vistas a la construcción de nuevas ideas y conocimientos sobre la innovación tecnológica y científica en el marco del desarrollo sustentable para las sociedades litorales.

\section{Area de estudio}

El sector de Guabún pertenece a la Comuna de Ancud ubicada en la zona norte de la isla de Chiloé. La caleta se encuentra a 24 kilómetros de la ciudad de Ancud y está localizada hacia el Océano Pacífico, por el borde costero occidental de la isla. Las viviendas están distribuidas en cadenas de pequeños cerros que las protegen de los vientos. En su ambiente hay una variedad de pequeñas playas donde predominan las dunas y la superficie es arenosa apareciendo rocas sólo en las orillas o zonas limítrofes de playas. Cuenta con 376 habitantes cuyas principales actividades son la pesca artesanal y la agricultura, las cuales históricamente se han conformado de acuerdo a un desplazamiento productivo desde las prácticas del campo hacia las del mar.

La actividad de agricultura incluye la crianza de animales y el cultivo de papas y hortalizas a lo cual se suma el trabajo en leña con fines domésticos. Las mujeres tienen instalados en sus hogares los huertos donde cosechan hortalizas, lo cual conforma un importante aspecto de la economía y alimentación familiar. Mediante buceo y/o recolección se extraen principalmente recursos tales como Luga (Gigartina skottsbergii), Llapín (Nothogenia afastigiata), Loco (Concholepas concholepas), Cochayuyo (Durvillaea antárctica), Luche (Porphyra columbina), Erizo de mar (loxechinus albus), Piure (Pyura chilensis), Lapa (Fissurella spp), entre otros.

Una de las principales características de esta caleta es su experiencia con el Área de Manejo y Explotación de Recursos Bentónicos (AMERB) para 
la especie Loco (Concholepas concholepas). Su éxito deriva de su sistema de prácticas que co-implica los saberes del campo en la administración de recursos del mar. El AMERB se encuentra en una de las playas del sector y su producción se destina únicamente al mercado internacional mediante la venta a empresas. Las otras actividades se destinan principalmente hacia el consumo familiar, la feria local de Ancud y la venta a intermediarios locales.

Las situaciones consideradas se contextualizan del siguiente modo en el ambiente local; a) la técnica para subir desde el fondo del mar a la superficie por parte de los buzos mariscadores incluye el uso de embarcaciones y la asistencia de compañeros que se quedan en la embarcación mientras el buzo desciende al mar. La antigüedad de esta práctica en Guabún y sus alrededores remite a la década de los ochenta y noventa, momento en el cual los precios de las algas tuvieron una subida (Boom) que generó un aprendizaje y concentración productiva en estas actividades (TAMAYO, 2007). Hasta el día de hoy estos saberes y prácticas permanecen en la memoria socio productiva de los habitantes del lugar como un complemento a las demás actividades.

b) Las prácticas en el Área de Manejo y Explotación de Recursos Bentónicos asociada a la especie Loco (Concholepas concholepas) remiten al contacto entre un habitante del sector de Guabún y un administrativo público que otorgó la información sobre los requisitos para adquirir el AMERB. Posteriormente, algunos habitantes formaron un sindicato de pescadores y buzos mariscadores llamado "Los Chonos de Guabún" que hasta el día de hoy se hace cargo de la administración del área. A su vez la selección del recurso Loco para ésta figura se debe a una historia de sobreexplotaciones a lo largo del país que motivó en gran medida la legitimación de estas figuras de administración en la Ley de Pesca (AVILÉZ y JERÉZ, 1999), c) la extracción del Llapín (Nothogenia afastigiata) y Luga (Gigartina skottsbergii) por parte de mujeres recolectoras y mariscadores habitantes del lugar se enmarca como una práctica que tiene su origen en el boom de algas al igual que en la primera situación.

\section{Resultados}

i) La subida desde el fondo marino a la superficie por los Buzos Mariscadores

Cuando un buzo está en lo profundo del mar y sube demasiado rápido, se produce un problema de descompensación por los rápidos cambios de presión. Al respecto, el conocimiento local de los mariscadores de Guabún ha establecido dos formas de subir a la velocidad adecuada. La primera de ellas 
consiste en subir siguiendo desde atrás la última burbuja que se libera desde la posición del buzo. La habilidad aquí, radica en vigilar la velocidad de subida con un indicador observable que, como tal, está dentro de la acción de subida, pues la última burbuja que va subiendo es visible al ojo del buzo $y$, por tanto, es posible para él regular su velocidad subiendo tras ella. La segunda consiste en subir en forma de zigzag método por el cual el buzo, mediante movimientos corporales, es capaz de desplazarse horizontalmente por el agua para así reducir la velocidad de subida.

Estas técnicas, conformadas en el tiempo solo por la recurrencia y el aprendizaje implicado en una práctica productiva, tiene las características de unir las habilidades del buzo a las condiciones específicas del medio (P. ej. las corrientes bajo el mar, el movimiento del agua y el peso del cuerpo). En este sentido, se trata de una técnica que emerge ajustada con el medio y asume más la forma de una habilidad práctica que la de una representación de conocimiento. La denominación de zigzag, expresa su comunicación como metáfora, pues los movimientos bajo el mar y las formas desplegadas son más complejos que la figura geométrica. De este modo, el vínculo que produce dicha metáfora soluciona el problema al modo de "un modelo que entrega "señales" para la activación de repertorios prácticos específicos apropiados en el contexto de la acción" (HORNBORG, 1996). Así, bajo ésta técnica verbalizada, las condiciones específicas del ambiente local en la situación de subida, se reorganizan bajo el marco de la experiencia y las habilidades adquiridas.

La metáfora utilizada del "zigzag" corresponde a una noción que no tiene su origen en los pescadores, así como la "burbuja", tampoco es una palabra construida especialmente para dicha situación. Ambas son objetos de conocimiento propios de la cultura occidental que han sido traspasados a los pescadores a través de capacitaciones. Lo interesante no es, en todo caso, el desplazamiento de significantes desde la sociedad mayor a las culturas locales (LONG, 2007), sino la nueva carga de diferencia de significado con que se han llenado esas categorías desde la experiencia del ambiente local. Lo principal no es, por tanto, aprender la representación del conocimiento -la metáfora como tal- sino que al conocer practicando se logra desplegar una habilidad esencialmente ensamblada a las condiciones de la situación y con ello se ajusta la orientación práctica hacia el medio ambiente (INGOLD, 1996). Como lo explicita el hecho anterior, la intención local al usar las palabras es comprender las condiciones de sus prácticas y traspasar las habilidades adquiridas a las nuevas generaciones. El injerto de nuevos significados hecho por la cultura local, por lo tanto, hace que dichas palabras tengan un rol efectivo y así un sentido legítimo dentro del universo local. 
Es importante considerar que la técnica del zigzag se combina con la técnica de la última burbuja, como queda de manifiesto en el siguiente relato:

Tiene que subir siempre en zigzag, porque sabe que no puede subir recto, (ya) trabajando dos horas o tres, y a más de doce metros de profundidad, no puede subir recto, ni rápido, porque si lo hace, las burbujas chicas van quedando debajo y esas burbujas (chicas) son las peligrosas que se meten en el cuerpo, él siempre tiene que subir en diagonal, nunca directo.

Ubicándonos en el mismo plano de la situación anterior, el conocimiento científico resuelve el problema mediante una línea imaginaria que va desde el fondo marino hasta la superficie. Una vez puesta la línea sobre la columna de agua se establecen puntos imaginarios de detención. El cálculo de la cantidad de puntos y el tiempo de detención en cada uno crece a medida que aumenta el tiempo en el fondo y la profundidad del mismo. Así, la fórmula sintetiza la relación entre las variables y entrega resultados según el tiempo y la profundidad. El proceso de reducción y simplificación realizado por el conocimiento científico se evidencia en que el buzo, al estar dentro de un ambiente dinámico, es imposible que se detenga a modo de imitar la precisión con la cual el modelo establece la resolución del problema. Parecería similar a la diferencia que había entre la metáfora y la acción del buzo, pero debemos considerar que mientras el conocimiento científico intenta activar razonamientos abstractos y una especie de imposibilidad de imitación, la metáfora en cambio activa repertorios y habilidades generando el acompasamiento corporal con el ambiente (HORNBORG 1996; INGOLD, 1996), lo cual hace pensar en una diferencia de efectos más que de naturaleza entre ambos tipos de conocimientos.

\section{ii) Las prácticas en un Área de Manejo y Explotación de Recursos Bentónicos}

Desde el lado científico-técnico estas figuras tienen el fin de realizar una explotación controlada de los recursos bentónicos, a través de un plan de manejo (MINECON, 1991) y además maximizar los beneficios económicos de su explotación (MONTECINOS, 2000).

El área llega a Guabún por conversaciones entre Pedro Pino y Juan Aedo, realizadas entre el 2000 y 2001 . En la perspectiva de Pedro, el vínculo con personajes externos en su quehacer político cotidiano es para establecer contactos beneficiosos. Las ideas del administrativo hicieron eco cuando planteó que el Estado entregaría zonas de mar a

Caderno eletrônico de Ciências Sociais, Vitória, v. 1, n. 1, p. 65-84. 
pescadores organizados. Los requisitos de formar esa organización para obtener un "pedazo de mar" generaron incredulidad y solo los más jóvenes se convencieron. Con el tiempo Guabún tiene su área bien cuidada, cada año rinde la cantidad esperada. La clave ha sido su economía familiar y el ser cultivadores en tierra, pues permitió comprender fácilmente el dejar un sector sin actividad antrópica un período al año. Además no se enfocaron solo en ella, la complementaron con otras actividades. En general, y al decir mismo de Juan Aedo, la singularidad de los habitantes de Guabún, así como el secreto de su área es que si bien coquetean con la modernidad, no se la tragan.

Al establecerse en una de las playas del sector de Guabún, el Área de Manejo y explotación de Recursos Bentónicos introduce, desde el inicio, un polígono geométrico irregular que delimita un trozo de mar concesionado a la organización de pescadores. Estas líneas imaginarias superpuestas sobre el mar ya definen un aspecto científico sobre el ambiente local. Además, implícitamente, esta figura incluye un funcionamiento propio dado por conocimientos científicos cuyo efecto modeliza los tiempos de las prácticas. Se trata de dos proposiciones científicas provenientes de las ciencias del mar; i) la idea del efecto positivo de la exclusión humana temporal en un sistema costero y ii) la explotación dentro de una zona geográfica delimitada, Ilamada en ecología puntos múltiples de estabilidad (MORENO et al., 1987).

De otro lado, la normativa que establece a las áreas de manejo, exige la presencia de una entidad técnica consultora introduciendo con esto una rutina de contacto entre biólogos marinos y pescadores artesanales con el objetivo de que cada año se mida el nivel de reproducción de las especies y a partir de ahí se defina la cantidad a extraer. La técnica utilizada por los biólogos consiste en cuadricular el polígono de área y tomar muestras al azar de los segmentos. Luego, mediante una fórmula es posible generalizar los resultados obteniendo un aproximado a la cantidad y los tamaños de las especies en el área. Finalmente, se calcula la cantidad de especies que puede ser extraída sin afectar su capacidad reproductiva.

No cabe duda de que el concepto eje para el sistema técnico es la explotación controlada, pero sin desconsiderar los lineamientos de este sistema, son los pescadores quienes por su continuidad y arraigo en el lugar pueden agregar y envolver con nociones sociales a las prácticas de la figura de administración ( $P$ ej. protección y defensa de los recursos, usos complementarios, valorización cultural del ecosistema o el comportamiento de otros factores integrantes de ese espacio bio-geográfico (POL, 2002; GARCÍA-ALLUT, 1999, 2003; REYES-GARCÍA y MARTÍ SANZ, 2007; GUERRA 
y SKEWES, 2008). Así, sobre la misma situación anterior, los pescadores de Guabún contextualizan el conocimiento técnico. Por ejemplo, trasladando habilidades aprendidas en el campo, como la habilidad de esperar los tiempos de cosecha realizando otra actividad, definen tanto el ritmo de trabajo, las cantidades a extraer y las complementaciones productivas para su área de manejo. Incluso muchas veces, sus ritmos y selección de cantidades, es menor a lo que los biólogos calculan como posible, de modo que, al tener en cuenta un ambiente local creado por la experiencia, ellos consideran otros factores que no se incluyen en el modelo de los biólogos, ampliando así los criterios de decisión.

Además, el conocimiento local de los pescadores artesanales ha definido prácticas y actividades (dentro) asumiendo la realidad del polígono del área de manejo; i) Cuando observan que el fondo marino presenta una distribución desigual de especies quedando piedras solitarias, consideran que no se está aprovechando bien el espacio y de no hacer algo se estaría dificultando la reproducción y crecimiento de las especies. Esto los ha llevado a redistribuirlas de forma homogénea para aprovechar mejor el área. ii) Puesto que observan las relaciones predatorias de otras especies sobre el recurso, se concentran en a) ubicar las especies en lugares provechosos para su alimentación y b) alejar las especies que se alimentan del recurso objetivo.

iii) La extracción de Algas de las recolectoras y buzos mariscadores

A partir de la diferenciación de conocimientos locales y científicos en torno a algunas algas es posible evidenciar otros aspectos sobre la posibilidad de vincular ambos conocimientos. La práctica de recolección de Luga (Sarcothalia crispata ) se aplica sobre las especies que quedan varadas sobre el arena y las rocas, producto de la poda natural que producen las mareas bajo el mar (JARAMILLO et al., 2006). Este sistema lo realizan, principalmente, mujeres. Se junta un grupo en una playa y van llenando sus brazos de algas y después lo llevan a la arena seca donde se esparce el producto formando un pequeño polígono cuadrado. Luego vuelven a sacar hasta que se acaba aquello que estaba varado o se encontraba flotando a media agua. En cambio, la práctica de buceo en profundidad implica el corte o poda artificial del alga que está en el fondo marino. Este sistema es utilizado en su mayoría por los hombres aunque hay mujeres que también lo utilizan.

Con respecto a la Luga, los habitantes tienen conocimiento sobre los tamaños mínimos para su extracción. El tamaño que debe tener para 
cortarla va de los 20 a $30 \mathrm{~cm}$. y el tamaño que se deja para que vuelva crecer va de los 10 a $5 \mathrm{~cm}$. Además, conocen los ritmos de crecimiento de las algas según las estaciones del año, de tal modo que las extraen solo en enero y febrero puesto que en invierno disminuye la velocidad de regeneración de la especie. En cada sector identifican las praderas naturales de algas y las rocas según la especie que vive en ellas.

Con respecto al Llapín (Nothogenia afastigiata), principalmente realizada por mujeres, se han identificado playas donde extraer y los días en que se demora su regeneración. Los períodos en que se debe dejar una playa sin actividad van de 9 a 10 días y la estación óptima de extracción ocurre en verano (diciembre a marzo). Además, los tiempos de las prácticas de extracción se ensamblan al ritmo físico-natural de las mareas beneficiando el proceso reproductivo de la especie. Cuando en una playa hacen un barrido general la espera de diez días sin actividad antrópica se trabaja en otras playas y rotando unas con otras permiten la reproducción del conjunto.

Sobre la misma situación, el conocimiento científico, basado en análisis de los procesos reproductivos de la especie, establece que es preferible determinar un tamaño mínimo identificado en $20 \mathrm{~cm}$. Además, establece que la cosecha óptima se lograría concentrando la actividad desde diciembre a marzo (AVILA et al., 2001). En este caso se observa un grado de similitud entre los resultados de la observación de los pescadores y las aseveraciones científicas.

\section{Discusión de resultados}

Consideradas simultáneamente las dos caras presentes en cada práctica relacionan los elementos a partir de un principio de organización. En el caso del conocimiento local, este principio está basado en organizar los elementos bajo una técnica que condensa habilidades adquiridas en el ambiente local. En la primera situación, la racionalidad local da contenido a las palabras "zigzag" y "última burbuja" desde un marco de referencia donde el universo biofísico no existe como representación mental de conocimiento. El conocimiento local solo repliega flujos de significados y los procesa desde la experiencia del lugar para la situación específica (ESCOBAR, 1996, 2009; GONZÁLEZ, 2009).

Al caracterizar algunos aspectos del conocimiento local, se observa que su conformación se establece siguiendo dos principios, i) el uso de la metáfora, como forma de comunicación de la experiencia, esto es, para enseñar y aprender habilidades muy específicas para situaciones concretas 
(HORNBORG 1996; INGOLD, 1996) y ii) el uso de la percepción visual como un elemento fundamental en la elaboración de conocimientos creativos con utilidad práctica inmediata (INGOLD, 1992; GARCÍA-ALLUT, 1999). En un plano simétrico, la metáfora, a diferencia de la racionalidad científica, envuelve y amplía la experiencia dejando posibilidades abiertas de conexión con otros conocimientos. En efecto, como se mostró en la situación de subida de los buzos, la técnica del zigzag se combina con la técnica de la última burbuja, lo cual se explica porque tanto el uso de la percepción visual como de la metáfora representan un marco común compartido y consensuado para la construcción de conocimientos y el despliegue de habilidades dentro del ambiente local.

En el caso del conocimiento científico componente de la situación de la subida de los buzos, el principio de organización es la experimentación externa que resulta en un modelo formal que luego es aplicado sobre una multiplicidad de factores que actúan en la acción y que por ende, lo desbordan. En este sentido la sola racionalidad científica implica descontextualización de aspectos concretos del ambiente local (LEFF, 2006). En vistas a su capacidad para comprender el ambiente, las líneas imaginarias de detención sobre la columna de agua se fundamentan en una "racionalidad sin percepción", es decir, en un argumento que al establecerse sobre instrumentos matemáticos -como la fórmula-, se cierra sobre sí misma aislándose del entorno de la acción. Impidiendo su combinación o fácil aprendizaje, la legitimidad de esta racionalidad produce que los buzos aun cuando tienen la experiencia suficiente, no obtienen su licencia de buzos por no pasar las pruebas matemáticas debido a su baja escolaridad. Así, se ven expuestos a multas constantes por una complicación que forma parte de las confusiones de la sociedad occidental con respecto a los tipos de conocimientos. Esto muestra la complejidad implícita en los contactos entre conocimientos (LONG, 2001) e induce, por lo demás, a pensar en que el propio lente de observación etnográfica tiene la responsabilidad en evidenciar dicha complejidad.

De otro lado, la situación de las áreas de manejo muestra que ambos tipos de conocimientos pueden complementarse satisfactoriamente en un espacio geográfico delimitado y en función de una población biológica definida. En esto, tanto el conocimiento local como el conocimiento científico pueden realizar sus roles complementándose (FLORIANNI, 2011). Incluso los propios recolectores de algas de las playas, al dejar algunos espacios geográficos específicos sin actividad para la reproducción de la especie y al utilizarlas de forma mutiespecífica, han llegado a las mismas proposiciones que desde ecología sustentan teóricamente a las áreas de manejo. Sin 
embargo en cuanto al acomodamiento entre ambas prácticas, el conocimiento de los pescadores es el que comienza a operar siguiendo los lineamientos del conocimiento científico. No es extraño pues los métodos científicos son rígidos y radicales en cuanto a la incertidumbre de encuentros o factores que existen en el contexto de aplicación (FLORIANNI, 2011). El conocimiento local se adapta como si se tratase de un medio práctico más, asumiendo incluso la figura de administración y los conteos de stocks realizados por los técnicos como guía y complemento.

Aun cuando no es el objetivo probar la validez de un conocimiento sobre otro, la tercera situación muestra que la experimentación y la experiencia, es decir, las observaciones locales y las científicas cada una con sus métodos, se diferencian, coinciden o se complementan (SALINAS, 2006; SCHUMANN, 2008). Sin embargo, se debe considerar que ambos tipos de conocimientos están más entremezclados de lo que podemos imaginar (LONG, 2001). Muchas de las proposiciones de los pescadores devienen desde contactos con actores externos así como también muchas de las aseveraciones de la ciencia tienen en su proceso de construcción el intercambio de conocimientos con habitantes de territorios locales (AGRAWAL, 1995). Estos dos aspectos, la coincidencia y el carácter combinado de los conocimientos, no significan que no sea necesario establecer diferencias, sobre todo si es con la finalidad de vincular y avanzar hacia nuevas formas de percibir y actuar con el medio habitado.

Considerando los modos en que se relacionan los conocimientos locales y científicos en el ambiente local, podemos aventurar que la vinculación entre ambos y la constitución de un tercero necesita nociones complejas para su entendimiento y constitución (OSTROM, 2000; BERKES y TURNER, 2005). Pues como se trata de un conocimiento fuertemente combinatorio, basado tanto en un nivel metafórico del significado como en una aproximación por razonamientos científicos, su imagen implica un movimiento que al tiempo que abre la experiencia hacia su conexión analógica con otros saberes, también se cierra y utiliza la experimentación. Su función sería establecer un vínculo que logre hacer el desenvolvimiento de la experiencia práctica al repliegue lógico del conocimiento científico. Se trata de un conocimiento marcado por i) la multiplicidad concreta de cada geografía local, ii) la metáfora como conector analógico y iii) la producción de variables y procedimientos de precisión. De éste modo, su finalidad es posibilitar que los habitantes de cada ambiente local puedan proyectar su futuro a través de una mirada aguda y al mismo tiempo reflexiva $y$ contextualizada. 
En concreto, con lo anterior establecido es un poco más evidente que el riesgo de la introducción de innovaciones solamente basadas en la racionalidad científica implicaría la fragmentación en la construcción sociocultural de los ambientes locales. En efecto, la separación entre experiencia y experimentación, implícita en la modernidad, tiene un efecto sobre las realidades locales. Al considerar dicha operacionalización como un discurso sus efectos se reflejan en la separación y desencuentros entre los actores científicos y los habitantes de sociedades litorales. Las diferencias de validez entre conocimientos científico y local están motivadas por este aspecto de la episteme moderna y se plasman en las prácticas y propuestas asociadas al desarrollo y la sustentabilidad de los ambientes locales.

\section{Conclusiones}

Las caletas de pescadores de la Isla de Chiloé han asimilado una acelerada y caótica modernización de la actividad pesquero artesanal en la cual las diferentes racionalidades puestas en juego han producido riesgos de descontextualización, aislamiento y pérdida de la capacidad de construcción social del futuro (MEDINA, 1998). En este contexto, el conocimiento local y el científico cobran relevancia no para validarse unos a otros sino más bien por la posibilidad que brinda su coexistencia para repensar el futuro de cada sociedad litoral a la luz de su potencialidad real y considerando la innovación como un producto asociado a sus particularidades. El enfoque de la antropología simétrica se presenta como un enfoque pertinente para abordar ambos tipos de conocimientos y para vincular a su vez, las perspectivas de la Etnoecología (REYES-GARCÍA y MARTÍ SANZ, 2007) y los Estudios Sociales de Ciencia y Tecnología (KREIMER, 2007), en la mira de construir alternativas de innovación basadas en la sustentabilidad de sociedades litorales vinculadas a ecosistemas específicos.

El sector de Guabún, ubicado en la zona noroccidental de la Isla de Chiloé, al ser una caleta de pescadores que al tiempo que realizan prácticas de campo y mar conservan rasgos tradicionales y asimilan aspectos de esta modernización, es un escenario ideal para el estudio de posibilidades alternativas de innovación a partir del análisis de los conocimientos locales y científicos. En el sistema de prácticas de este asentamiento, se destacan tres situaciones que ilustran de buena forma las asimilaciones y los impactos recíprocos entre ambos tipos de conocimientos. Así, en la situación de subida del buzo a la superficie, el conocimiento local actúa por medio de la metáfora y la percepción visual re-significando con los contenidos locales las palabras adquiridas por medio de la educación formal. En el área de manejo 
y explotación de recursos bentónicos se destaca la complementación de ambos conocimientos, con una asimilación por parte del conocimiento local sobre aquellos que el conocimiento científico establece en asociación al marco normativo de la actividad. Finalmente, las prácticas de recolección muestran que existen acercamientos entre las observaciones del conocimiento local y científico al tiempo que no es siempre posible desentrañar exactamente el origen de un tipo de conocimiento por su naturaleza esencialmente combinatoria.

Considerando lo anterior, las posibilidades de un conocimiento innovador remiten a diferenciar para luego relacionar, vincular e incorporar ambos tipos de conocimientos (VESSURI, 2004; KREIMER, 2007). Las situaciones prácticas capturadas por una etnografía de carácter hologramático, interpretadas a la luz de una perspectiva simétrica, permiten analizar de forma simultánea ambos tipos de conocimientos como formando parte de la construcción sociocultural de un mismo ambiente local. Con ello es posible pensar en un tercer conocimiento que al tiempo que incluya a los anteriores permita unir la separación moderna entre experiencia y experimentación que se refleja en la fragmentación de los ambientes locales (HOPENHAYN, 1993; MEJÍA, 2008; FLORIANNI, 2011). El hecho simple de que la destrucción de significados y la destrucción de ecosistemas sean dos aspectos del mismo proceso (HORNBORG, 1996), implica al menos tres necesidades para las preocupaciones por el desarrollo de sociedades vinculadas a ecosistemas locales; i) La necesidad de trabajar de forma interdisciplinaria y en contacto con los habitantes y sus experiencias del ambiente, ii) La necesidad de un conocimiento que implique la participación de los distintos actores que hoy son parte de estos ambientes y iii) La necesidad de re-considerar a la innovación más que como el avance en productividad, como la conformación de un conocimiento compartido en vistas al futuro sustentable.

\section{Bibliografía}

AGRAWAL, A. 1995. Dismantling the divide between indigenous and western knowledge. Development and Change, 26 (3): 413-439.

ÁLAREZ, C. 2011. La política pesquero artesanal en Chile desde una aproximación antropológica: la mesa público-privada en la zona contigua, Regiones de Los Lagos y Aysén. Facultad de filosofía y humanidades, Escuela de Antropología, Universidad Austral de Chile.

ARIAS, L. 2009. Interdisciplinariedad y triangulación en Ciencias Sociales. Diálogos, Revista Electrónica de Historia, 10 (1):117-136 
AVILA, M., CACERES, J., CANDIA, A., PLAZA, H., SAN MARTIN, R., GONZALES, J. y OTROS. 2001. Investigación y Manejo de praderas de Luga en la $X$ y XI Regiones. FIP-IT/99-21, Instituto de Fomento Pesquero (IFOP), Fondo de Investigación Pesquera.

AVILÉZ, O. y G. JERÉZ. 1999. Desarrollo de capacidades de gestión local: gestión sustentable de recursos marinos bentónicos en caletas de la IV Región. Ambiente y Desarrollo, 4: 6-10.

BERKES, F. y TURNER, N. 2005. Conocimiento, aprendizaje y la flexibilidad de los sistemas socioecológicos. Gaceta Ecológica, 77: 5-17.

BUSTAMANTE, R. y J. C. CASTILLA. 1987. The shellfishery in Chile: An analysis of 26 years of landings (1960-1985). Biología Pesquera, 16:7997.

BUSTOS E., GONZALEZ A. y F. PONCE. 1991. Repoblación de recursos bentónicos: Mecanismos de recuperación de poblaciones sobreexplotadas. Revista Pacifico Sur, 19:35-37.

CANALES, M. 2006. Metodologías de investigación social, introducción a los oficios. Santiago: LOM Editores.

CASTILLA J. 1990. Clase magistral: La importancia y proyección de la investigación de ciencias del mar en Chile. Revista de Biología Marina, Valparaíso, 25(2): 1-18.

CEBALLOS, M. 2010. Análisis de la ocupación territorial y manejo de algas en las caletas de Guabún y Pupelde incorporando los saberes tradicionales. Comuna de Ancud, Región de los Lagos. Memoria para optar al título de geógrafo. Facultad de Arquitecutra y Urbanismo, Escuela de Geografía, Universidad de Chile.

DIAZ, A. 2008. Procesos sociales y lógicas productivas en la relación entre Pescadores Artesanales, Estado y Mercado. Tesis para optar al título de antropólogo y al grado de licenciado en Antropología, Universidad Austral de Chile, Escuela de Antropología, Valdivia.

DIAZ DE RADA, A. 2006. El taller del etnógrafo. Materias de metodología de la etnografía. Disponible en: [www.uned.es/dpto asyc/594036/etnografo.pdf].

ESCOBAR, A. 1996. La invención del tercer mundo. Bogotá: Norma.

ESCOBAR, A. 2009. El lugar de la naturaleza y la naturaleza del lugar: ¿Globalización o Postdesarrollo? Recuperado el 15 de enero de 2013, Disponible en: [http://www.unc.edu/ aescobar/text/esp/lugardenaturaleza.pdf].

EYLES, J. 1998. Los métodos cualitativos en geografía humana: bases teóricas y filosóficas y aplicaciones prácticas. En: García, A. (Ed.), 
Métodos y técnicas cualitativas en geografía social, (33-44), Barcelona: Oikos-tau.

FLORIANNI, N. 2011. Saberes y prácticas de territorios agroecológicos. Punta Grossa: Editora UEPG.

GAJARDO, C. 2011. Saberes y prácticas pesquero-artesanales en el contexto de la modernización. Cotidianidad y desarrollo en las caletas de Guabún y Puñihuil, Provincia de Chiloé. Facultad de filosofía y humanidades, Escuela de Antropología, Universidad Austral de Chile.

GARCÍA-ALLUT, A. 1999. Compartición del conocimiento tradicional y científico para una gestión más adecuada de las pesquerías. Etnográfica, 3 (2), 109-331.

GARCÍA-ALLUT, A. 2003. La pesca artesanal, el cambio y la patrimonialización del conocimiento. Boletín del Instituto Andaluz del Patrimonio Histórico (44), 74-83.

GIDDENS, A. 1991. Modernity and Self-Identity. Self and Society in the Late Modern Age. Cambridge, Polity Press.

GONZÁLEZ, F. (2009) Desarrollo humano sustentable local. Revista POLIS, Vol. 8, No 22: 53-66.

GUERRA, D., y SKEWES, J. C. 2008. ¿Vernacularización, Hibridación, Enajenación o Patrimonialización? Conserva (12), 5-37.

HENRÍQUEZ, R. 2010. Legislación pesquera y paisaje litoral: los pescadores artesanales de la localidad de Pucatrihue, comuna de San Juan de la Costa, Chile. Tesis para optar al grado de licenciado en Antropología y al título de Antropólogo, Universidad Austral de Chile, Escuela de Antropología, Valdivia.

HOPENHAYN, M. 1993. El Humanismo Critico como campo de saberes sociales en Chile. En J. J. Brunner, M. Hopenhayn, T. Moulian, \& L. Paramio, Paradigmas de conocimiento y práctica social en Chile (203278). Santiago: FLACSO.

HORNBORG, A. 1996. La ecología como semiótica. En Descola, P. y Pálsson, G. (coord.) Naturaleza y Sociedad, Perspectivas Antropológicas, (pp. 60-80).

IBÁÑEZ, C., PIZARRO, R., y VIAL, R. 2001. La Privatización de los Recursos del Mar. Análisis de Políticas Públicas (11), 1-24.

INGOLD, T. 1992. Culture and the perception of the environment. En Croll,

E. y D. Parkin (eds.), Bush base-forest farm: culture, environment and development. Londres: Routledge.

INGOLD, A. 1996. El forrajero óptimo y el hombre económico. En Descola, P. y Pálsson, G. (coord.) Naturaleza y Sociedad, Perspectivas Antropológicas, (pp. 60-80). México: Siglo Veintiuno. 
JARAMILLO, E., DE LA HUZ, R., DUARTE, C., y CONTRERAS, H. 2006. Depósitos de algas varadas y artrópodos macrofaunales en playas de arena de la costa de Chile. Rev. Chilena de Hist. Nat. , 79 (3), 337-351.

JóCILES, M. I. 1999. Las técnicas de investigación en antropología social: mirada antropológica y proceso etnográfico. Gazeta de Antropología, 15.

KNORR-CETINA, K. 1981. The Manufacture of Knowledge: An Essay on the Constructivist and Contextual Nature of Science. Oxford: Pergamon Press

KREIMER, P. y H. THOMAS. 2004. Un poco de reflexividad o ¿de dónde venimos? Estudios sociales de le ciencia y la tecnología en América Latina. En Kreimer, P. et al. (eds.), Producción y uso social de conocimientos, Estudios de sociología de la ciencia en América Latina, (11-90), Bernal: Universidad Nacional de Quilmes.

KREIMER, P. 2007. Estudios sociales de la ciencia y la tecnología en américa latina: ¿Para qué?, ¿Para quién?, Redes, 13(26): 55-64.

LANDER, E. 2000. Ciencias Sociales: Saberes coloniales y eurocéntricos. En E. Lander(comp.), La colonialidad del saber: eurocentrismo y ciencias sociales. Perspectivas latinoamericanas. Buenos Aires: CLACSO.

LATOUR, B. 1993. We Have Never Been Modern, London: Harvester Weatsheaf

LEFF, E. 2001. Epistemología Ambiental. Sao Paulo: Cortez Editora.

LEFF, E. 2002a. Saber Ambiental. Racionalidad, sustentabilidad, complejidad, poder. México: Siglo XXI Editores.

LEFF, E. 2002b. Ética, Vida, Sustentabilidad. Serie Pensamiento Ambiental Latinoamericano, 5.

LEFF, E. 2006. La ecología política en América Latina. Un campo en construcción, Revista Polis de la Universidad Bolivariana, 2 (5).

LINDÓN, A. 2006. La espacialidad de la vida cotidiana: hologramas socioterritoriales de la cotidianidad urbana. En J. Nogué y J.Romero (Eds.). Las otras geografías (pp. 425-446). Valencia: Tirant Le Blanch.

LINDÓN, A. 2007. Los imaginarios urbanos y el constructivismo geográfico: los hologramas espaciales. Eure, 33(99): 31-46.

LONG, N. 2001. Antropología del desarrollo, perspectivas del actor. Londres y Nueva York: Routledge.

MARCUS, G. 2001. Etnografía en/del sistema mundo. El surgimiento de la etnografía multilocal. Alteridades, 11(22): 111-127.

MARIN, A. y BERKES, F. 2010) Network approach for understanding smallscale fisheries governance: The case of the Chilean coastal comanagement system. Marine Policy (34): 851-858. 
MARIN, W. 2007. Caleta los Bronces, Cambio de una cultura competitiva extractiva a una sustentable asociativa. Tesis presentada para obtener el grado de Magíster en Antropología y Desarrollo, Universidad de Chile, Departamento de Antropología, Santiago.

MEDINA, J. 1998. La Prospectiva humana y social: alternativa de la nueva generación para américa latina? Ciclo de conferencias sobre conocimiento, globalización y territorio.

MEJIA, J. 2008. Epistemología de la investigación social en América Latina. Desarrollos en el siglo XXI. Cinta de Moebio, 31, 1-13.

MINECON. 1991. Ley General de Peca y Acuicultura (No 18891/1991). Valparaiso.

MONTECINOS M. 2000. Las Áreas de Manejo y Explotación de Recursos Bentónicos: Génesis, desarrollo, implementación e implicancias para la conservación de los recursos bentónicos en Chile. Tesis para optar al grado de Licenciado en Biología Marina. Universidad Austral de Chile.

MORALES, H. L. 1986. La modernización de las pesquerías chilenas. Impactos sociales y ecológicos. Ambiente y Desarrollo, 2 (2), 33-46.

MORENO C., GODOY C., VILLOUTA E. y M. LEPEZ. (1987). Explotación de los recursos bentónicos litorales: Una alternativa derivada de la protección de áreas. En Arana (ed.): Manejo y desarrollo pesquero. Escuela de Ciencias del Mar. Universidad Católica de Valparaíso. Pp. 51-58

MORIN, E. 1998. Epistemología de la Complejidad. En D. Schnitman, Nuevos paradigmas, cultura y subjetividad (págs. 421-442). Buenos Aires: Páidos.

NANDY, A. 1988. Science, Hegemony and violence: a requiem for modernity. Oxford University Press.

NEIRA, P. 2005. Las comunidades de pescadores artesanales frente a la modernización: el caso de la caleta Queule. Memoria para optar al título de Antropóloga Social, Universidad de Chile, Departamento de Antropología, Santiago.

OSTROM, E. 2000. Diseños complejos para manejos complejos. Gaceta ecológica. 54: 43-58.

POL, E. 2002. City-Identity-Sustainability (CIS). Environment and Behavior, 34(1).

PEÑA, J. 1996. Regulación pesquera en Chile: una perspectiva histórica. Cuadernos de Economía, 33 (100), 367-395.

REYES-GARCIA, V. y N. MARTI-SANZ. 2007. Etnoecología: punto de encuentro entre naturaleza y cultura. Revista ecosistemas, 16(3), 4554. 
SALINAS, O. 2006. Evaluación del conocimiento tradicional de pescadores artesanales de Caleta Hornos, IV Región, acerca del recurso "loco" (Concholepas concholepas). Tesis de Licenciatura para optar al Título de Biólogo Marino, Universidad Católica del Norte, Departamento de Biología Marina.

SCHUMANN, S. 2008. ¿Colaboración o Colisión? La relación entre los pescadores artesanales y sus consultoras técnicas, y su relevancia para las áreas de manejo en Chile. Recuperado el 27 de Junio de 2010, de Federación Regional de Pescadores Independientes y Afines de la Octava Región. Disponible en: [www.ferepabiobio.cl/xpdinam/db/archivos/.../Colaboracion o colision. pdf].

TAMAYO, M. 2007. Reconstrucción histórica de las estrategias adaptativas en comunidades de pescadores artesanales. Dos casos en la décima región, provincia de Chiloé. Tesis de grado para optar al título de Antropólogo y el grado de Licenciado en Antropología, Universidad Austral de Chile, Escuela de Antropología, Valdivia.

THER RÍOS, F. (comp.). 2003. Niveles y Perspectivas de Investigación en Ciencias Sociales. Diseños Investigativos. Osorno: CEDER.

THER RÍOS, F. 2006. Complejidad territorial y sustentabilidad: notas para una epistemología de los estudios territoriales. Horizontes Antropológicos 25: 105-115.

THER RÍOS, F. 2008. Las sociedades litorales como sistemas de prácticas y saberes tradicionales y científicos: la caleta como modelo de imaginarios pesqueros y dinámicas de uso y apropiación de recursos marinos. Proyecto Fondecyt 1080665.

VALDERRAMA, J. 2010. Situación de las figuras de administración pesquera sobre los territorios costeros en Chile. Sustentabilidad y dinámicas socioculturales en 3 caletas de la comuna de Ancud, X Región. Facultad de Arquitectura y Urbanismo, Escuela de Geografía, Univ. de Chile.

VESSURI, H. 2004. La hibridización del conocimiento. La tecnociencia y los conocimientos locales a la búsqueda del desarrollo sustentable. Convergencia, 11 (35): 171-191.

VILLORO, L. 2008. Creer, saber, conocer. México: Editorial Siglo XXI.

VIVEIROS DE CASTRO, E. 2002. O nativo relativo, Revista Mana, 8 (1), 113142. 